\title{
Maritime wireless broadband communication system based on TVWS
}

\author{
Yonghui Zhang ${ }^{1,}$, Z Zhenjia Chen, Fang Dong, Baodan Chen \\ ${ }^{1}$ College of Information Science \& Technology, Hainan University, 58 Renmin Ave., \\ Haikou, Hainan, 570228, China \\ aemail: zhyhemail@163.com
}

Keywords: TVWS; Mesh Network; ROS; Socket; H264

\begin{abstract}
TV White Spaces (hereinafter referred to as the "TVWS") is the radio spectrum has not been used, is assigned to using in broadcast television. TVWS has the advantages of high transmission distance, strong penetrating ability, not easy to be absorbed by water, large amount of transmission unit data, etc., low spectrum occupancy rate in maritime, is very suitable for maritime wireless network coverage. In this paper, the maritime communication system based on TVWS uses the wireless mesh network as the network architecture. Communication access point(hereinafter referred to as the "AP") based on the software router system (hereinafter referred to as the "ROS"). TVWS is used as the distance communication spectrum between APs. The AP implements the data exchange between WIFI and TVWS, and provides Ethernet wired access and WIFI wireless access for the terminal equipment. The communication system uses the Socket transmission protocol to set up the end to the end connection, realizes the functions of GPS, character communication, picture transmission, audio communication, video communication and camera monitoring, etc., using H264 video compression format to achieve smooth video communication.
\end{abstract}

\section{Introduction}

In current, communication devices in the sea using a single sideband shortwave radio, very high frequency(VHF) wireless phone, ultrashort wave(USW) interphone, cellular phones, satellite phones and other. These communication methods have advantages and disadvantages: the principle of the single sideband shortwave radio communication is reflected by the atmosphere. It has long communication distance, has lower costs than satellite phone, is terminal equipment maintenance is convenient. But this way has communication cover blind area, vulnerable to interference like weather, low confidentiality; VHF wireless phones can communicate about 20 nautical miles and only for the ship to shore and ship between voice communication; USW interphone is mainly used for voice communication at close range between the ships, unable to realize broadband communication; cellular handset communication costs low, but cellular base station signal coverage distance is limited, usually only in the offshore available. At present, commercial fishing, shipping, emergency rescue, or coast guard communications are based on the satellite and deploy mesh network in maritime[1]. For ship and land communication, the ship carrying mobile terminal is connected to the maritime wireless mesh network first, and then through the ship borne satellite gateway access satellite mobile network[2]. The satellite mobile communication is suitable for the ocean shipping, but the terminal configuration, maintenance and communication costs are relatively high. Small and medium vessels at sea are often unable to buy such expensive satellite communications and navigation equipment. In order to integrate the heterogeneous wireless and wired networks, people proposed that the different paths of the ship and the ship, the ship and the land traverse through the VoIP system. Using double IP address and dual SIP address to manage mobile user and VoIP session. VoIP system selects the best path to reduce the data transmission link to reduce the data transmission delay and reduce the cost of data exchange[3]. The system mentioned in this paper realizes the construction of TVWS and WIFI heterogeneous network, realizes the long-distance wireless broadband communication in maritime, will not produce any communication costs. Each AP can be used as a network access gateway to share the network resource. This is a maritime wireless communications solution. 


\section{Operating frequency}

TVWS is in the $30-300 \mathrm{MHz}$ VHF and $300-3000 \mathrm{MHz}$ UHF, which is the gold band in the radio frequency. In maritime, such gold band has not been utilized. Compared to other more high frequency segments, TVWS has excellent radio propagation characteristics as mentioned above. Internationally, TVWS is used as the preferred band for LTE deployment. At the beginning of LTE network deployment, TVWS has a vital role to the rapid coverage of LTE network, which is favored by the major mobile communication operators. Therefore, the system in this paper is proposed to use the TVWS as the operating frequency band between the communication APs in maritime. In the process of experiments, we use $700 \mathrm{MHz}-800 \mathrm{MHz}$ as the test frequency band.

\section{System network}

Maritime communication requires a flexible networking mode, which must be used as the basis of the network. The ship can set up its own wireless local area network to carry out simple communication. Maritime communication also requires that the network has good reliability and stability, but also need to have the ability to manage. In this paper, we build a wireless Mesh network base on rapid spanning Tree Protocol(hereinafter referred to as the "RSTP"). Mesh network has the characteristics of self organization, self configuration and self repair. These features make the wireless network in the sea can be free and flexible organization, simple, easy to maintain. The network has a very high scalability and reliable service quality, and the cost is relatively low. Network terminal can be any type of wireless equipment. The AP in the network as a gateway for different types of internal network or bridge[4].

ROS is a low cost, high performance router system. Most existing routing systems have already started using the ROS function. The system is suitable for schools, enterprises, small ISP-access providers, Internet cafes, Internet community and other network equipment[5]. In this paper, communication APs are based on ROS, each AP in the network as a client, and the AP devices are composed of a point to point network structure. The AP is used to communicate with each other AP in the $700 \mathrm{MHz}-800 \mathrm{MHz}$ frequency band by 802.11 standards.

When the distance between two APs is beyond the range of the single AP's signal coverage, two APs can automatically select the intermediate AP as the repeater, thus expanding the communication distance. When there are multiple APs can choose as a repeater or need to go through multiple relay route, the RSTP can provide path delay calculation for the communication between APs, and choose the optimal path. The network structure of point to point wireless transmission is shown in Figure 1.

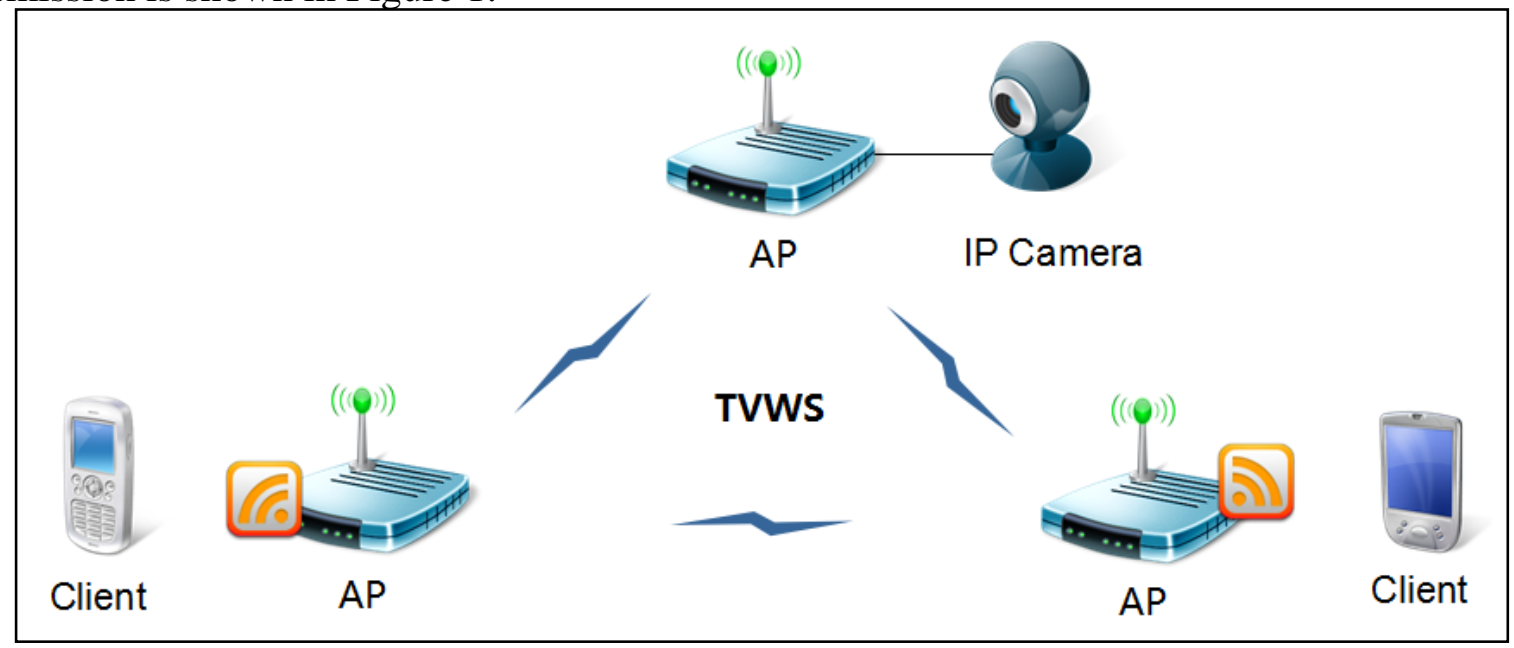

Fig.1. The network structure of point to point wireless transmission

\section{Software implementation}

Software applications use socket communication protocol, to achieve the end to the end 
communication. First, the terminal transmits the broadcast to the network through the multicast technology. Second, the other terminal in the network receives the data packet, then parse out the IP address and user information. At last, terminal according to the IP address and the corresponding service port to establish the service connection, carries on the data exchange. Software implementation process as shown in Figure 2.

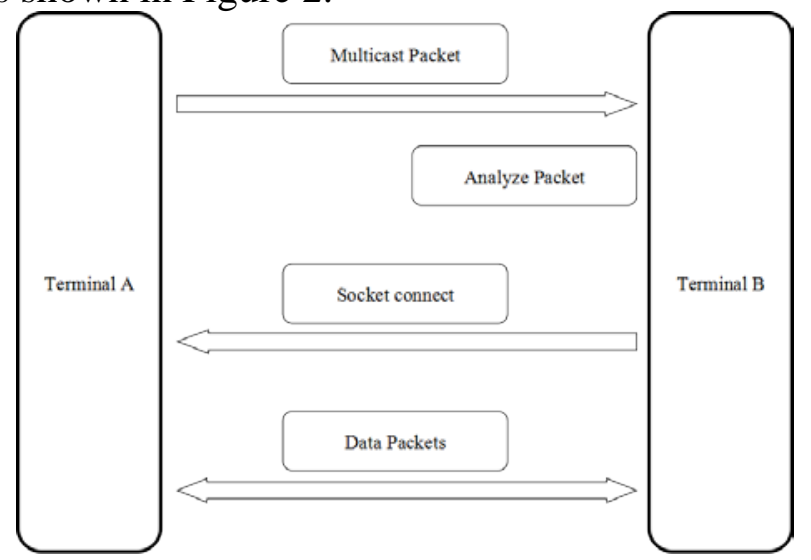

Fig.2. Software implementation process

Multicast implementation of a broadcast form of one-way network connection. The sending end sets the destination host address in network to transmit the broadcast packet. The whole group of all the hosts(terminal devices) can receive the data. Multicast technology can effectively solve the problem of single point sending and receiving, and can realize the efficient data transmission, which can save network bandwidth, reduce network load, improve data transmission efficiency and reduce the possibility of congestion in backbone network.

Socket is used to describe the IP address and port, is a communication link handle, can be used to realize the communication between different virtual machines or different computers. In order to realize the application layer and network layer communication in the software, we uses the socket API for the software platform. In design patterns, socket is actually a facade pattern. Socket interface to hide the complexity of the TCP/IP protocol. For users, socket is a set of simple interfaces. Socket self organize data to achieve the specified protocol. According to the use of sockets, we can divide the device into a server end and a client end. The socket of the terminal opens the local server port in the form of the server end, and the process is waiting for the connection. As a client, the target is to connect to the server. The client socket sends a connection request to the server socket by the server's IP address and port. The server socket receives a connection request from the client, then the process is converted from the waiting connection state to the response request state. The server side sends the description of the socket to the client. After the client confirms the description, the connection is established.

The communication AP is assigned a static IP address for each access terminal and locate every mobile terminal. Cross between APs, the system uses the communication AP as the route, and uses the socket transport protocol to carry on the communication between the end to the end. The system supports the characters, pictures, audio and video communication between mobile terminals. The transmitting end turns data into a data stream and writes the data stream into the pipeline. The receiving end receives various types of data streams according to the service port, and converts the data stream into the corresponding data information according to the data type. In order to optimize the transmission efficiency of video communication, we use H264 video compression format to process the video data in the mobile terminal. H264 is used to improve the quality of video encoding [6]. Compared to MPEG and H263, it can improve the efficiency of about 40\% to 60\%[7]. A frame data compression of a video to preserve the integrity of the image information. Compression frame data using JPEG method can only reach $60 \%$ of the compression rate. The H264 frame data is stored in the inter frame or the intra frame prediction image information. Compared to the ordinary video compression method, H264 video compression format has about 95\% compression ratio. In this system, the data volume of the terminal transmits a second 320x240 specifications video is only about 25KB. To a large extent reduce the stress of data transmission. 


\section{Test results}

According to the characteristics of the communication system, we carry out a series of related field tests. In the process of the long distance wireless communication between the terminals, the main test content is the communication quality of the system. A fixed AP is built on the roof of the college, which is used as the repeater of the APs in our laboratory. In addition, we set the mobile AP near the college about 600 meters. We have three APs for video communication quality testing. AP layout is shown in Figure 3. AP1 is installed fiberglass omnidirectional antenna gain of 8dBi. AP2 and AP3 are installed sucker antenna gain of 12dBi. Wherein, all APs are using a dual antenna configuration, one responsible for receiving another responsible for sending.

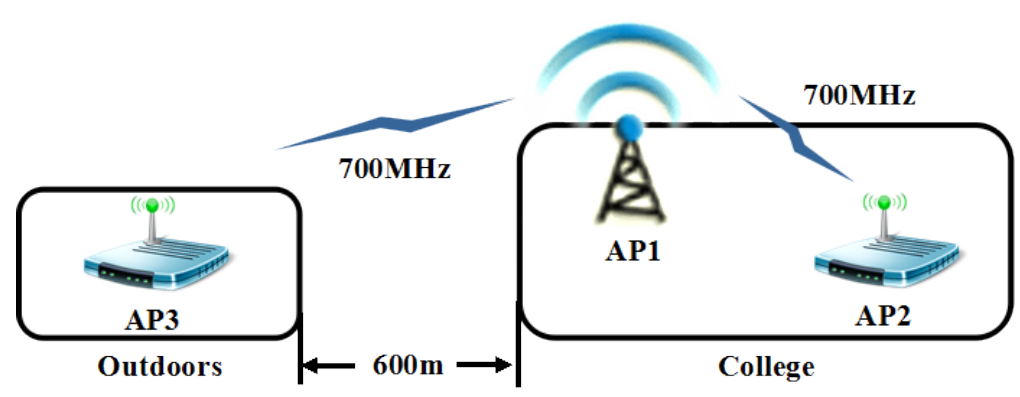

Fig.3. AP layout

Test data are shown in Table 1.

Table 1. Communication delay data for AP3 and other APs

\begin{tabular}{ccc} 
Destination AP & AP1 & AP2 \\
\hline Average delay & $337 \mathrm{~ms}$ & $212 \mathrm{~ms}$ \\
Packet loss rate & $10 \%$ & $12 \%$ \\
Transmission rate & $2.1 \mathrm{Mbps}$ & $1.9 \mathrm{Mbps}$ \\
\hline
\end{tabular}

The test data for the AP3 and other nodes are shown in Figure 4 and Figure 5.

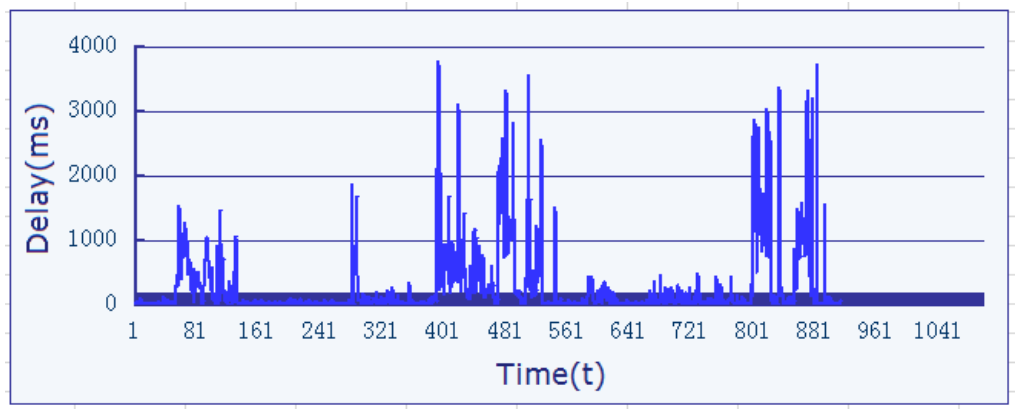

Fig.4. Time delay of communication between AP3 and AP1 


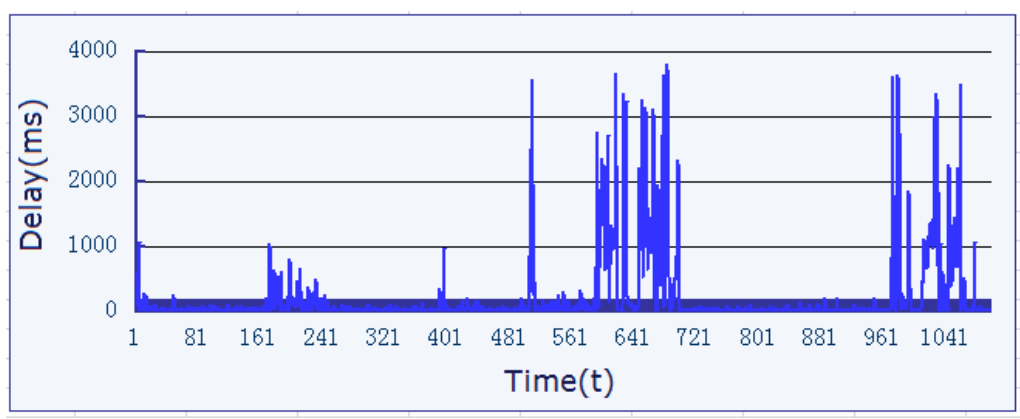

Fig.5. Time delay of communication between AP3 and AP2

In the process of testing, the occlusion or other factors in the signal transmission path will affect the performance of the network, and increase the link delay. Overall, the performance of the network is basically stable, the average delay is lower, and broadband communication can be realized. The increase of the number of link hops, such as the link of node AP3 to node AP2, can lead to the increase of packet loss rate.

Next, we carry out remote communication testing. We choose two experimental sites in Haikou Bay to build communication APs, the linear distance is about $8 \mathrm{~km}$. The distribution of the experimental sites in Haikou Bay is shown in Figure 6. APs are installed fiberglass omnidirectional antenna gain of $8 \mathrm{dBi}$, and use a single antenna configuration, an antenna is responsible for sending and receiving.

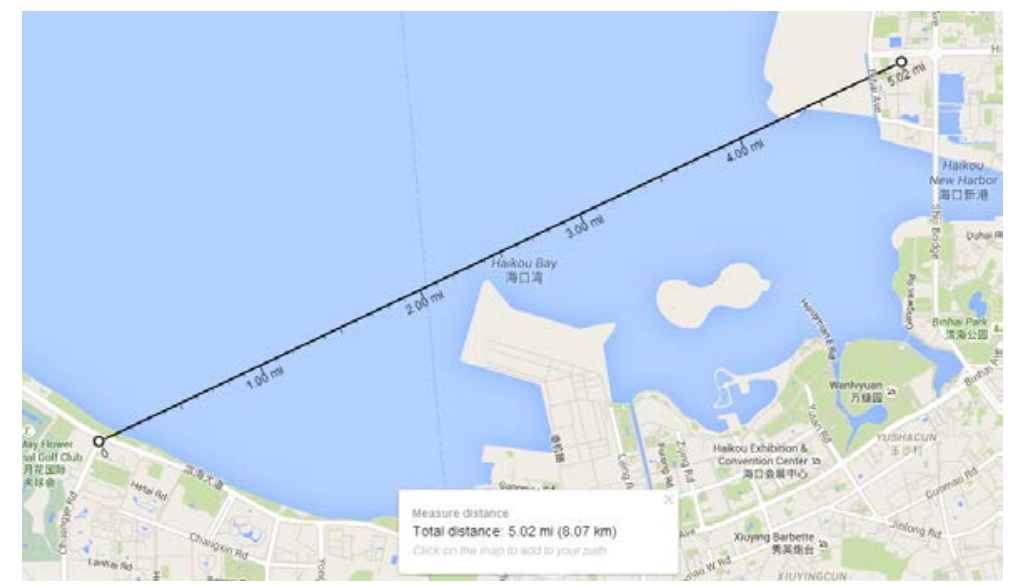

Fig.6. The distribution of the experimental sites in Haikou Bay

We test the communication quality between two APs. After the test, the total packet loss rate is $1 \%$, the average delay is $72 \mathrm{~ms}$, the transmission rate is $1.6 \mathrm{Mbps}$. AP delay test data is shown in Figure 7.

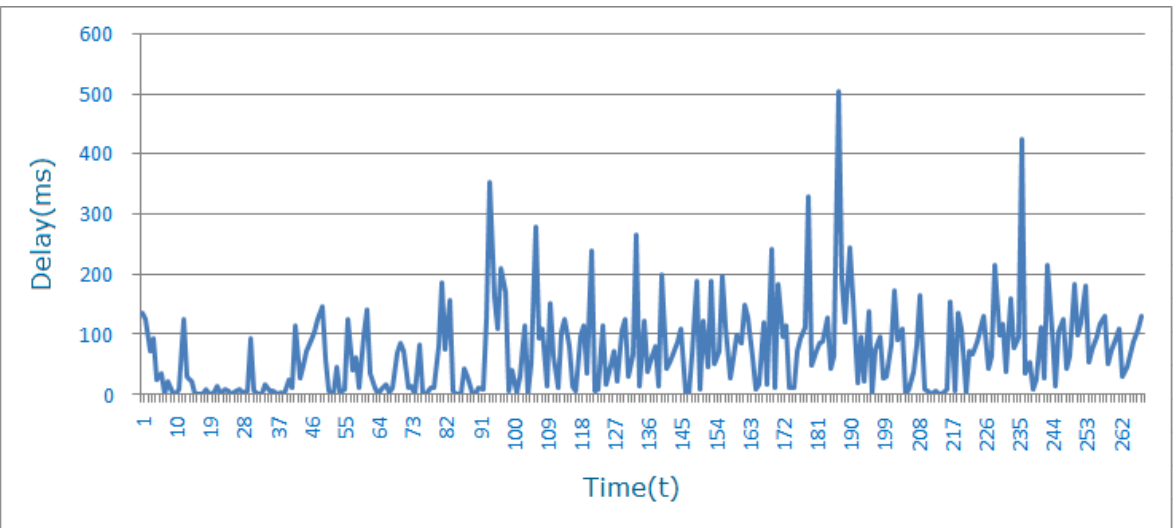

Fig.7. Time delay between two communication APs in Haikou Bay

Then carry on the test of the terminal remote video communication. The delay test data is shown in Figure 8 .The total packet loss rate is $2 \%$, the average delay is $72 \mathrm{~ms}$. Video communication effect is very good, no caton phenomenon appears. 


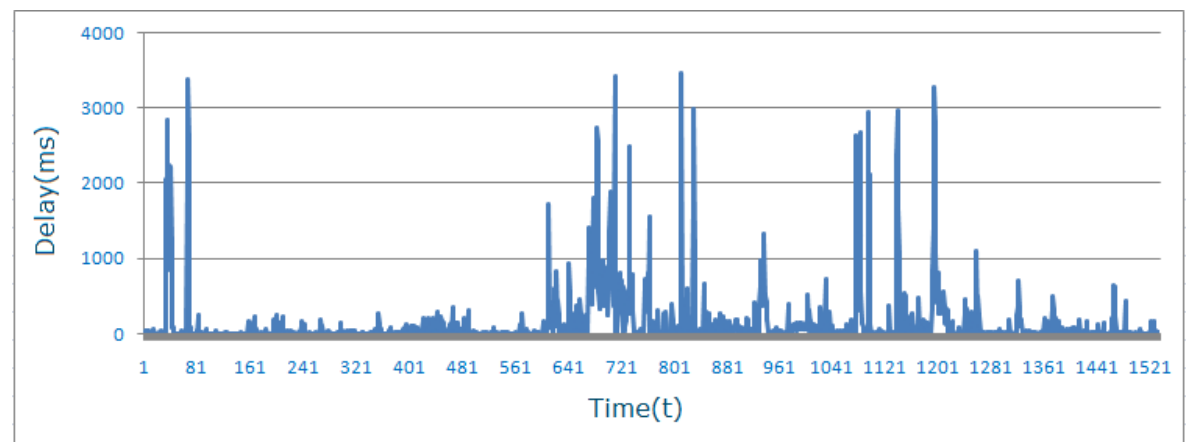

Fig.8. Time delay of the terminal remote video communication in Haikou Bay

The test environment has been carefully designed to be close to the maritime environment. Test results are consistent with the design expectations. Because of the problem of antenna matching, the response delay and packet loss rate can be improved. We found that when the AP's signal is blocked by buildings, the AP is difficult to be connected to other APs. In addition, the low speed of the mobile AP connection and data transmission is not obvious.

\section{Conclusion}

With the shortage of spectrum resources, the radio frequency spectrum provides more usable frequency band, but the application is also facing a variety of challenges. The use of the blank spectrum shows its commercial value, and the bottleneck of its development is the lack of reliable networking mode and specific communication scheme. This system uses the software router to realize the heterogeneous network of TVWS and WIFI 2.4GHz. According to the characteristics of TVWS to realize the remote wireless broadband communication, this system achieved rapid networking, strong mobility, low price of maritime wireless communication scheme. This scheme not only can be used in maritime communications, but also can provide low-cost technical means for remote areas of wireless broadband connection, for example in the field of open field of gobi or grassland.

\section{Acknowledgement}

In this paper, the research was sponsored by the National Natural Science Foundation of China (Grant No. 61561018), the Application Technology Research and Development and Demonstration of Hainan Province (Grant No. ZDXM2015104) and the Collaborative Innovation Fund Project of Tianjin University - Hainan University and the International S\&T Cooperation Projects of China (Grant No. 2015DFR10510).

The corresponding author is Yonghui Zhang.

\section{References}

[1] M. Manoufali, H. Alshaer, P.-Y. Kong, and S. Jima, “Technologies and networks supporting maritime wireless mesh communications," in 6th Joint IFIP Wireless and Mobile Networking Conference (WMNC), pp. 1 - 8, Apr. 2013.

[2] Wencai Du, Zhengxin Ma, Yong Bai, Chong Shen, Youling Zhou, Baodan Chen, “Integrated Wireless Networking Architecture for Maritime Communications,” pp.134-138, SNPD 2010.

[3] Yong Bai, Wencai Du, “VoIP Services for Ocean Fishery Vessels over Integrated Wireless and Wireline Networks,” pp.3461-3465, PIMRC 2013.

[4] HWANG Ren-junn, HSIAO Yu-kai, "A Secure and Reliable Routing Protocol for Wireless Mesh Networks,” pp.466-475, Shanghai Jiaotong Univ. (Sci.), 2014. 
[5] Jun Cheng, Honghua Wu, "The Application Of The PPPOE For Network Security Management Using RouterOS,” pp.569-571, ICCDA 2010.

[6] Richardson, I.E.G.: H.264 and MPEG-4 Video Compression:Video Coding for Next-generation Multimedia. Wiley, New York (2003)

[7] ISO/IEC DIS 13818 - 2, MPEG-2 video coder 